\title{
Erratum: Non-collinear generation of angularly isolated circularly polarized
} high harmonics

Daniel D. Hickstein, Franklin J. Dollar, Patrik Grychtol, Jennifer L. Ellis, Ronny Knut, Carlos Hernández-García, Dmitriy Zusin, Christian Gentry, Justin M. Shaw, Tingting Fan, Kevin M. Dorney, Andreas Becker, Agnieszka Jaroń-Becker, Henry C. Kapteyn, Margaret M. Murnane and Charles G. Durfee

Nature Photonics 9, 743-750 (2015); published online 20 September 2012; corrected online 7 October 2015; corrected after print 15 December 2015

In the version of this Article originally published the blue dashed line was mislabelled in the legend in Fig. 3d and the label should have read $i \mathbf{E}_{\text {vert }}$. This has now been corrected in the online versions of the Article. 